SHORT REPORT

\title{
Primary lateral sclerosis presenting parkinsonian symptoms without nigrostriatal involvement
}

\author{
N Mabuchi, H Watanabe, N Atsuta, M Hirayama, H Ito, H Fukatsu, T Kato, K Ito, G Sobue
}

J Neurol Neurosurg Psychiatry 2004;75:1768-1771. doi: 10.1136/jnnp.2003.035212

We encountered three patients with primary lateral sclerosis (PLS) showing bradykinesia, frozen gait, and severe postural instability, as well as slowly progressive spinobulbar spasticity. Cranial magnetic resonance (MR) imaging showed precentral gyrus atrophy. Central motor conduction was markedly prolonged or failed to evoke a response. Positron emission tomography (PET) showed significant reduction of $\left[{ }^{18} \mathrm{~F}\right]$ fluoro-2-deoxy-D-glucose uptake in the area of the precentral gyrus extending to the prefrontal, medial frontal, and cingulate areas. No abnormalities were seen in the nigrostriatal system with PET using $\left[{ }^{18} \mathrm{~F}\right]$ fluorodopa or $\left[{ }^{11} \mathrm{C}\right]$ raclopride or with proton MR spectroscopy. Thus, widespread prefrontal, medial, and cingulate frontal lobe involvement can be associated with the parkinsonian symptoms in PLS.

$\mathrm{P}$ rimary lateral sclerosis (PLS), a form of motor neurone disease (MND), is a rare non-familial neurodegenerative disorder. PLS is characterised by slowly progressive spinobulbar spasticity reflecting exclusive involvement of upper motor neurones. ${ }^{12}$ Spastic dysarthria and paraparesis, emotional lability, and pseudobulbar palsy are major clinical features of PLS.

On rare occasions, MND may give rise to bradykinesia and motor fatigue. These probably arise from generalised spasticity. However, freezing of gait and severe postural instability suggest more widespread system involvement and have not been well described. We present three patients with PLS showing these two parkinsonian-like features in addition to spinobulbar spasticity and bradykinesia. We investigated these symptoms in the patients using positron emission tomography (PET) including $\left[{ }^{18} \mathrm{~F}\right]$ fluoro-2-deoxy-D-glucose (FDG), $\left[{ }^{18} \mathrm{~F}\right]$ fluorodopa (F-dopa), and $\left[{ }^{11} \mathrm{C}\right]$ raclopride (RACLO), as well as proton magnetic resonance spectroscopy $\left({ }^{1} \mathrm{H}-\mathrm{MRS}\right)$.

\section{PATIENTS AND METHODS}

\section{Patients}

The three patients, all men, fulfilled Pringle's diagnostic criteria for PLS. ${ }^{1}$ None had a familial history of PLS, and all showed a slowly progressive course.

\section{Patient 1}

Slowness and clumsiness of the right hand became evident at the age of 43 years. Spasticity involved the right leg, and caused spastic dysarthria by age 44 . Soon afterward, the spasticity spread to all four limbs. At age 45 , he showed a severely frozen gait and occasional falling, especially backward. He also showed severe akinetic posturing and postural instability. He was unable to walk unaided by age 51 . Speech gradually became unintelligible because of severe spastic dysarthria, and he was unable to speak aloud at age 53 .

\section{Patient 2}

Frozen gait with initial hesitation developed at age 62, followed by cramping in both legs during sleep at age 64 . He began to experience speech disturbance with spastic dysarthria and bouts of unprovoked laughing and crying. He showed severe postural instability with occasional falling, especially backward, as well as akinesia. He could not stand up or initiate walking smoothly. He also showed facial hypokinesia with reduced blinking. All symptoms showed gradual progression.

\section{Patient 3}

Postural instability and gait disturbance were present by age 68 . He noted left arm clumsiness, and paucity of movement gradually worsened and progressed to akinesia. He developed spastic dysarthria at age 69. He showed occasional falling, especially backward. All symptoms showed gradual progression.

\section{Methods}

PET study

PET studies were performed using a ECAT EXACT HR47 (CTI/ Siemens, Knoxville, TN) and HEADTOME-IV (Shimadzu, Kyoto, Japan) for three patients with F-dopa, FDG, and two of the three patients with RACLO in three dimensional acquisition mode, which yielded 47 simultaneous planes, with an axial full width half maximum resolution of $4.8 \mathrm{~mm}$ and an inplane resolution of $3.9 \times 3.9 \mathrm{~mm}$. The protocol for F-dopa and FDG injection has been described previously. ${ }^{3}$ RACLO was injected at $494 \mathrm{MBq}$, and scanning consisted of 19 time frames for a total of 64 minutes. Photo data were presented with three dimensional stereotactic surface projection (3D-SSP) as normalised for the whole brain. 3D-SSP was performed as described by Minoshima et al. ${ }^{5}$ Data were normalised to global activities. The patients' datasets were compared individually with the normal database by calculating a z score on a pixel-to-pixel basis, and were displayed in a 3D-SSP display for visual inspection.

\footnotetext{
${ }^{1} \mathrm{H}-\mathrm{MRS}$ study

${ }^{\mathrm{l}} \mathrm{H}$-MRS was performed with a 3.0-T system (Bruker, Germany) using a standard head coil with circular polarisation. The spectroscopic volume of interest was placed in the putamen $\left(1 \mathrm{~cm}^{3}\right) \cdot{ }^{1} \mathrm{H}-\mathrm{MR}$ spectra were acquired using a point-resolved spectroscopy sequence with chemical-shift selective water suppression. The metabolic ratios $\mathrm{N}$-acetylaspartate (NAA)/creatine ( $\mathrm{Cr}$ ), and choline containing phospholipids (Cho)/Cr were determined as semiquantitative values. Data were compared with those of 18 age matched control subjects. The protocol was as previously described. ${ }^{6}$
}

Abbreviations: FDG, $\left[{ }^{18} \mathrm{~F}\right]$ fluoro-2-deoxy-D-glucose; F-dopa, $\left[{ }^{18}\right.$ F]fluorodopa; PET, positron emission tomography; ${ }^{1} \mathrm{H}-\mathrm{MRS}$, proton magnetic resonance spectroscopy; MND, motor neurone disease; PLS, primary lateral sclerosis; RACLO, [ $\left.{ }^{11} \mathrm{C}\right]$ raclopride 
Table 1 Characteristics of the three patients with primary lateral sclerosis

\begin{tabular}{|c|c|c|c|}
\hline & Patient 1 & Patient 2 & Patient 3 \\
\hline $\begin{array}{l}\text { Age at examination, } \\
\text { (years)/sex }\end{array}$ & $53 / M$ & $66 / M$ & $72 / M$ \\
\hline Duration of illness (years) & 10 & 4 & 4 \\
\hline Spastic dysarthria & ++ & ++ & ++ \\
\hline Babinski sign (left/right) & $\begin{array}{l}\text { Present/ } \\
\text { present }\end{array}$ & $\begin{array}{l}\text { Present/ } \\
\text { present }\end{array}$ & $\begin{array}{l}\text { Present/ } \\
\text { present }\end{array}$ \\
\hline Spasticity in limbs & ++ & ++ & ++ \\
\hline Bradykinesia & ++ & ++ & ++ \\
\hline Postural instability & +++ & ++ & ++ \\
\hline Frozen gait & ++ & ++ & - \\
\hline $\begin{array}{l}\text { Decrease of amplitude in } \\
\text { finger and foot tapping }\end{array}$ & ++ & ++ & ++ \\
\hline Rigidity & - & - & - \\
\hline Tremor & - & - & - \\
\hline
\end{tabular}

\section{RESULTS}

The clinical features of the three patients are shown in table 1. General physical examinations were normal in all patients. On neurologic examination, all three patients showed spastic dysarthria. Ocular movements were saccadic, but covered a full range. Muscle power was essentially normal (patient 1 , all limbs normal; patient 2, Medical Research Council grade $4 / 5$ in the legs and 5/5 in the arms; patient 3, 4/5 in the left biceps and iliopsoas muscles, but otherwise normal). Muscle tone was greatly increased in the limb flexor muscles but not in the extensors, this was appreciated best upon rapid movement. The jaw jerk reflex and deep tendon reflexes in the legs were brisk, and plantar responses were bilaterally extensor in all patients. No wasting, fasciculations, amyotrophy, sensory impairment, or cognitive dysfunction was seen in any patient.

With respect to parkinsonian symptoms, slowness and fatiguing of voluntary movement, poverty of movement, loss of facial expression, and reduction of blinking were observed in all patients. Finger tapping and foot tapping were performed slowly, with gradual reduction in amplitude. All patients showed a shuffling, short stepped gait with backward falling en bloc without corrective steps when equilibrium was momentarily upset. The posture of patient 1 was stooped. Patient 2 showed some emotional lability. Patients 1 and 2 showed freezing, particularly at initiation of gait, although ability to move otherwise was relatively preserved. Kinésie paradoxale was not observed. Levodopa was not effective in any patient.

Haematological and serum biochemistry tests gave normal results. Tests for human $\mathrm{T}$ lymphocytotropic virus $\mathrm{l}$ and syphilis were negative in all patients. Cerebrospinal fluid test results were normal except in patient 2, who had slightly elevated protein $(65 \mathrm{mg} / \mathrm{dl})$. MR images of the cervical spine were normal except for non-specific degenerative changes without spinal cord compression. Cranial MR images showed generally mild cortical atrophy including the precentral gyrus in all patients. ${ }^{28}$ Nerve conduction studies were normal. Concentric needle electromyography (EMG) showed only focal neurogenic change in the right first dorsal interosseous muscle (patients 1 and 2), left gastrocnemius (patient 2), and right triceps (patient 1) without fulfilling revised El Escorial criteria. ${ }^{9}$ In all patients, motor evoked potentials showed markedly increased central motor conduction time or failed to evoke responses in the lower limbs. ${ }^{2} 10$

FDG-PET showed significantly decreased glucose uptake in the area of the precentral gyrus (table 2, fig 1). The reduced glucose uptake extended to the medial frontal lobe including the prefrontal area (patients 1 and 2) and the anterior cingulate cortex (patients 2 and 3). In addition, patient 3 showed significantly low glucose uptake in the medial posterior parietal lobe. No significant reduction of glucose utilisation was observed in the basal ganglia in any patient. In addition, F-dopa and RACLO PET showed no significant reduction of uptake in the putamen and caudate nucleus compared with control subjects. NAA/Cr and Cho/Cr values in the putamen were also within the normal range.

\section{DISCUSSION}

We studied three patients with PLS who, in addition to the expected bulbospinal spasticity, presented poverty of movement, loss of facial expression with reduced blinking, frozen gait with initial hesitation, gradual reduction in finger and foot tapping rate and amplitude, and severe postural instability that mimicked parkinsonism. They also all showed symmetric spasticity, hyperreflexia, extensor plantar responses, and spastic dysarthria. No fasciculations, muscle

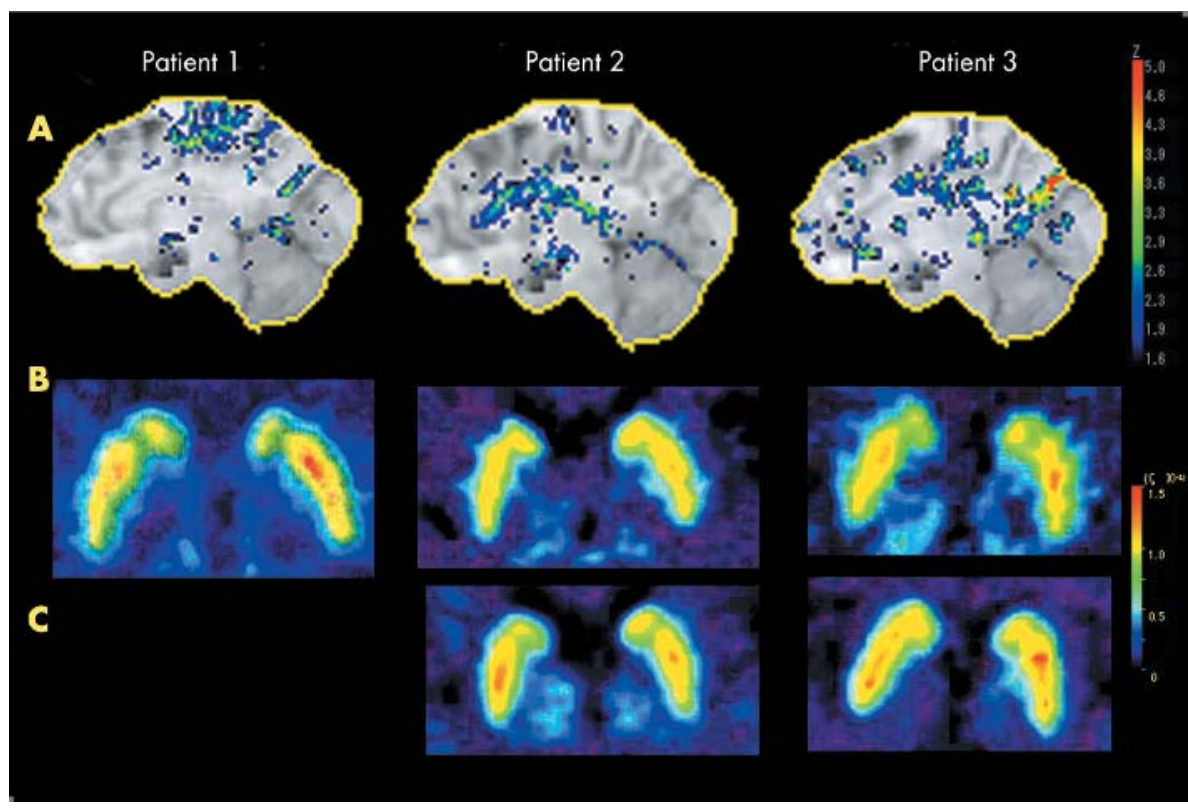

Figure 1 Positron emission

tomographic (PET) images are shown for three patients with primary lateral sclerosis (PLS). (A) An FDG-PET image (right medial view) shows significant reduction of FDG uptake from the precentral cortex to the supplementary motor cortex (patient 1); from the precentral cortex to the anterior cingulated cortex (patient 2); and near the precentral gyrus (patient 3). (B) No uptake reductions were observed in the basal ganglia with F-dopa (all patients) or (C) [ [ $\left.{ }^{11} \mathrm{C}\right]$ raclopride (patients 2 and 3) 
Table 2 Electrophysiological, MRI, and 'H-MRS features in the three patients with primary lateral sclerosis

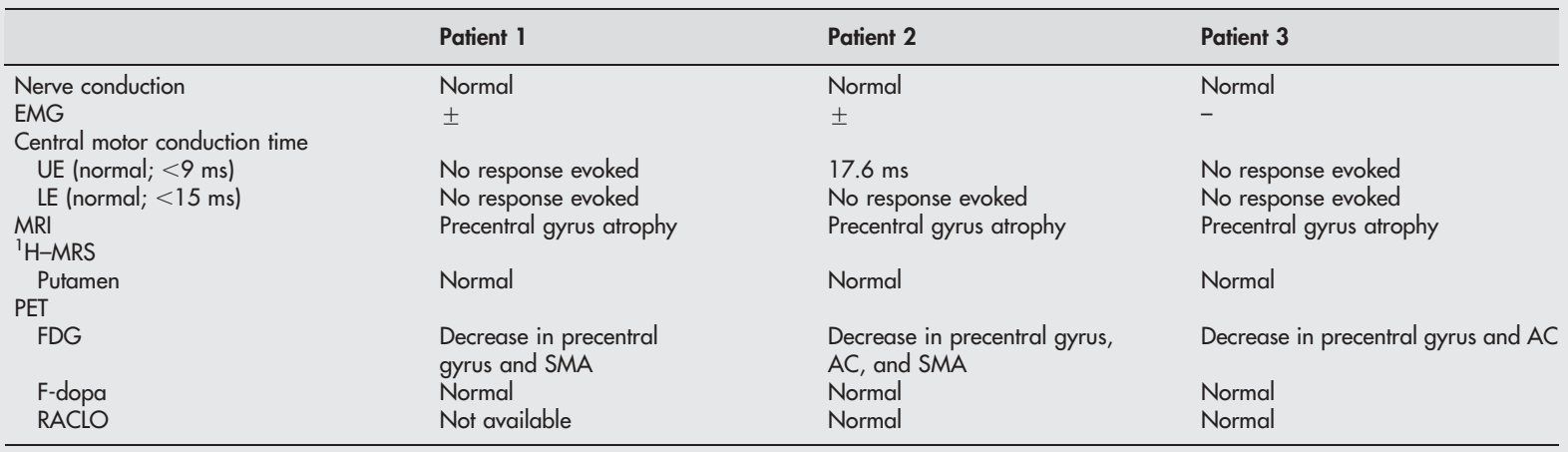

\pm , neurogenic change present but not fulfilling revised El Escorial criteria; -, no abnormality.

$\mathrm{AC}$, anterior cingulate cortex; EMG, electromyography; FDG, $\left[{ }^{18} \mathrm{~F}\right]$ fluoro-2-deoxy-D-glucose; F-dopa, $\left[{ }^{18} \mathrm{~F}\right]$ fluorodopa; UE, upper extremities; LE, lower extremities; MRI, magnetic resonance imaging; ${ }^{1} \mathrm{H}-\mathrm{MRS}$, proton magnetic resonance spectroscopy; $\mathrm{PET}$, positron emission tomography; RACLO, [ $\left.{ }^{11} \mathrm{C}\right] \mathrm{raclopride}$; SMA, supplementary motor area.

atrophy, or weakness were noted, and the sensory and other systems were uninvolved. No patient fulfilled the diagnostic criteria for amyotrophic lateral sclerosis, ${ }^{9}$ but all fulfilled the criteria for PLS proposed by Pringle et al. ${ }^{1}$ In the symptomatology of PLS, facial hypokinesia and lack of animation have been reported, ${ }^{1}$ but patients presenting not only generalised bradykinesia but also parkinsonian-like frozen gait with initial hesitation and severe postural instability have not been well described.

Several neurodegenerative diseases including multiple system atrophy, frontotemporal dementia, ${ }^{11}$ and progressive supranuclear palsy show pyramidal tract signs and parkinsonism. These signs have also been reported concomitant with amyotrophic lateral sclerosis and Parkinson's disease. ${ }^{12}{ }^{13}$ However, the present patients did not show symptoms clearly indicative of these diseases. Furthermore, characteristic signal abnormalities and morphologic changes were not observed on MR images. None of the three patients exhibited nigrostriatal system involvement on PET (FDG, F-dopa, and RACLO) and ${ }^{1} \mathrm{H}$-MRS images. In addition, there was no improvement with dopaminergic medication. Considering all observations, the parkinsonian symptoms in our three patients were unlikely to have been caused by nigrostriatal involvement.

Frozen gait and severe postural instability including falling backward en bloc without corrective steps are commonly reported in pathologically proved progressive supranuclear palsy, vascular parkinsonism, and advanced Parkinson's disease. ${ }^{14}{ }^{15}$ Although the pathophysiological background of such symptoms is unclear, several PET studies have emphasised the causal importance of medial frontal lobe involvement. ${ }^{16-18}$ In addition, pathological examination of patients showing frozen gait suggested that the frontal lobe as well the basal ganglia would represent essential lesion sites. $^{14}{ }^{15} 19$ The most striking observation in the present study was that FDG-PET uptake showed significant reductions extending from the precentral gyrus to the medial frontal cortex in all patients, including the supplementary motor area and anterior cingulate cortex. In contrast, the basal ganglia of all three patients were well preserved according to PET and ${ }^{1} \mathrm{H}$-MRS. Considering these observations together, it would seem that not only generalised spasticity but also widespread frontal lobe impairment was responsible for the parkinsonian symptoms in our patients with PLS. ${ }^{75} 19$

Recent MRI and PET studies have demonstrated widespread involvement of the precentral gyrus as well as ventrolateral, prefrontal, and anterior cingulate cortices in PLS. ${ }^{20}$ Although spasticity may mask the presence of other clinical features, precise radiological study is expected to shed further light on the pathophysiology and symptomatology of PLS. We believe that parkinsonian symptoms in PLS without nigrostriatal involvement can result from frontal lobe involvement.

\section{Authors' affiliations}

N Mabuchi, H Watanabe, N Atsuta, M Hirayama, H lto, G Sobue, Department of Neurology, Nagoya University Graduate School of Medicine, Nagoya, Japan

H Fukatsu, Department of Radiology, Nagoya University Graduate School of Medicine, Nagoya, Japan

T Kato, K Ito, Department of Biofunctional Research, National Institute for Longevity Sciences, Obu, Japan

Competing interests: none declared

Correspondence to: Dr G Sobue, Department of Neurology, Nagoya University Graduate School of Medicine, Nagoya, 466-8550 Japan; sobueg@med.nagoya-u.ac.jp

Received 5 January 2004

In revised form 16 March 2004

Accepted 24 March 2004

\section{REFERENCES}

1 Pringle CE, Hudson AJ, Munoz DG, et al. Primary lateral sclerosis. Clinical features, neuropathology and diagnostic criteria. Brain 1992; 1 15:495-520.

2 Kuipers-Upmeijer J, de Jager AEJ, Hew JM, et al. Primary lateral sclerosis: clinical, neurophysiological, and magnetic resonance findings. I Neurol Neurosurg Psychiatry 2001;71:615-20.

3 Ito K, Nagano-Saito A, Kato T, et al. Striatal and extrastriatal dysfunction in Parkinson's diseases with dementia. A 6-[18F]fluoro-L-dopa PET study. Brain 2002; 125:1358-65.

4 Arahata $Y$, Hirayama $M$, leda $T$, et al. Parieto-occipital glucose hypometabolism in Parkinson's disease with autonomic failure. J Neurol Sci 1999;163:119-26.

5 Minoshima S, Frey KA, Koeppe RA, et al. A diagnostic approach in Alzheimer's disease using three-dimensional stereotactic surface projections of fluorine-18-FDG PET. J Nucl Med 1995;36:1238-48.

6 Watanabe $H$, Fukatsu $H$, Katsuno $M$, et al. Multiple regional $1 \mathrm{H}-\mathrm{MR}$ spectroscopy in multiple system atrophy: $\mathrm{NAA} / \mathrm{Cr}$ reduction in pontine base as a valuable diagnostic marker. J Neurol Neurosurg Psychiatry 2004;75:103-9.

7 Zhai P, Pagan F, Statland J, et al. Primary lateral sclerosis. A heterogeneous disorder composed of different subtypes? Neurology 2003;60:1258-65.

8 Smith CD. Serial MRI findings in a case of primary lateral sclerosis. Neurology 2002;58:647-9.

9 Brooks BR, Miller RG, Swash M, et al. El Escorial revisited: revised criteria for the diagnosis of amyotrophic lateral sclerosis. Amyotroph Lateral Scler Other Motor Neuron Disord 2000; 1:293-9.

10 Le Forestier N, Maisonobe T, Piquard A, et al. Does primary lateral sclerosis exist? A study of 20 patients and a review of the literature. Brain 2001;124:1989-99. 
11 Mochizuki A, Komatsuzaki Y, Iwamoto $\mathrm{H}$, et al. Frontotemporal dementia with ubiquinated neuronal inclusions presenting with primary lateral sclerosis and parkinsonism: clinicopathological report of an autopsy case. Acta Neuropathol 2004;107:377-80.

12 Qureshi Al, Wilmot G, Dihenia B, et al. Motor neuron disease with parkinsonism. Arch Neurol 1996:53:987-91.

13 Desai J, Swash M. Extrapyramidal involvement in amyotrophic lateral sclerosis: backward falls and retropulsion. I Neurol Neurosurg Psychiatry 1999:67:214-16.

14 Yanagisawa N, Hayashi R, Mitoma H. Pathophysiology of frozen gait in parkinsonism. Gait disorders. Adv Neurol 2001;87:199-207.

15 Demirkiran M, Bozdemir H, Sarica Y. Vascular parkinsonism: a distinct heterogeneous clinical entity. Acta Neurol Scand 2001;104:63-7.
16 Kikuchi A, Takeda A, Kimpara T, et al. Hypoperfusion in the supplementary motor area, dorsolateral prefrontal cortex and insular cortex in Parkinson's disease. J Neurol Sci 2001;193:29-36.

17 Escola L, Michelet Th, Macia F, et al. Disruption of information processing in the supplementary motor area of the MPTP-treated monkey. A clue to the pathophysiology of akinesia? Brain 2003;126: 95-114.

18 Cunnington R, Egan GF, O'Sullivan JD, et al. Motor imagery in Parkinson's disease: A PET study. Mov Disord 2001;16:849-57.

19 Factor SA, Jennings DL, Molho ES, et al. The natural history of the syndrome of primary progressive freezing gait. Arch Neurol 2002;59:1778-83.

20 Le Forestier N, Maisonobe T, Spelle L, et al. Primary lateral sclerosis: further clarification. J Neurol Sci 2001;185:95-100.

\section{Clinical Evidence-Call for contributors}

Clinical Evidence is a regularly updated evidence-based journal available worldwide both as a paper version and on the internet. Clinical Evidence needs to recruit a number of new contributors. Contributors are healthcare professionals or epidemiologists with experience in evidence-based medicine and the ability to write in a concise and structured way.

Areas for which we are currently seeking authors:

- Child health: nocturnal enuresis

- Eye disorders: bacterial conjunctivitis

- Male health: prostate cancer (metastatic)

- Women's health: pre-menstrual syndrome; pyelonephritis in non-pregnant women

However, we are always looking for others, so do not let this list discourage you.

Being a contributor involves:

- Selecting from a validated, screened search (performed by in-house Information Specialists) epidemiologically sound studies for inclusion.

- Documenting your decisions about which studies to include on an inclusion and exclusion form, which we keep on file.

- Writing the text to a highly structured template (about 1500-3000 words), using evidence from the final studies chosen, within 8-10 weeks of receiving the literature search.

- Working with Clinical Evidence editors to ensure that the final text meets epidemiological and style standards.

- Updating the text every six months using any new, sound evidence that becomes available. The Clinical Evidence in-house team will conduct the searches for contributors; your task is simply to filter out high quality studies and incorporate them in the existing text.

- To expand the topic to include a new question about once every 12-18 months.

If you would like to become a contributor for Clinical Evidence or require more information about what this involves please send your contact details and a copy of your CV, clearly stating the clinical area you are interested in, to Klara Brunnhuber (kbrunnhuber@ bmigroup.com).

\section{Call for peer reviewers}

Clinical Evidence also needs to recruit a number of new peer reviewers specifically with an interest in the clinical areas stated above, and also others related to general practice. Peer reviewers are healthcare professionals or epidemiologists with experience in evidence-based medicine. As a peer reviewer you would be asked for your views on the clinical relevance, validity, and accessibility of specific topics within the journal, and their usefulness to the intended audience (international generalists and healthcare professionals, possibly with limited statistical knowledge). Topics are usually 1500-3000 words in length and we would ask you to review between 2-5 topics per year. The peer review process takes place throughout the year, and our turnaround time for each review is ideally 10-14 days.

If you are interested in becoming a peer reviewer for Clinical Evidence, please complete the peer review questionnaire at www.clinicalevidence.com or contact Klara Brunnhuber (kbrunnhuber@bmigroup.com). 